International Journal of Diabetology \& Vascular Disease Research (JJDVR)

ISSN:2328-353X

\title{
Prevention of Diabetic Nephropathy in Children and Adolescents: How Effective are the Current Strategies?
}

SN Uwaezuoke

Review Article

Paediatric Nephrology Firm, Department of Paediatrics, University of Nigeria Teaching Hospital, Ituku-Ozalla, Enugu, Nigeria.

\begin{abstract}
This paper aims to review the risk factors for diabetic nephropathy (DN) and the effectiveness of the current strategies for its prevention. Type 1 diabetes mellitus (T1DM) is the predominant form of diabetes in children and adolescents, but the prevalence of Type 2 diabetes mellitus (T2DM) in these age groups is currently increasing worldwide. One of the major challenges of T1DM is the development of $\mathrm{DN}$ among other microvascular complications. DN evolves over a long period of time starting from microalbuminuria and progressing to end stage renal failure. While microalbuminuria in children is reversible and may not evolve to end-stage renal failure, macroalbuminuria inevitably progresses to end-stage renal failure irrespective of any known treatment. Several modifiable and non-modifiable risk factors are well documented in the literature but addressing a few are critical in the prevention of DN.

For instance, tight glycemic control and intensive control of hypertension have significant impact on prevention and progression of DN. From several studies, tight glycemic control has been shown to decrease the risk of microvascular disease in both T1DM and T2DM. Thus, poor glycemic control is critical in the etiology of DN. While tight glycemic control in patients with T1DM reduces the incidence of microalbuminuria and the progression from microalbuminuria to macroalbuminuria, there is overwhelming evidence to show that antihypertensive treatment with angiotensin converting enzyme (ACE) inhibitors is important in both preventing and treating microalbuminuria, and thus preventing progression to overt $\mathrm{DN}$. Dietary protein restriction is also an adjunct strategy in retarding the progression of DN. Other novel therapeutic strategies have been recently tried and found potentially effective. Research is ongoing to establish the clinical efficacy and usefulness of some of these new agents in future.
\end{abstract}

Keywords: Childhood Diabetes; Diabetic Nephropathy; Microvascular Complications; Risk Factors; Prevention.

\section{*Corresponding Author:}

Dr SN Uwaezuoke,

Paediatric Nephrology Firm, Department of Paediatrics, University of Nigeria Teaching Hospital, Ituku-Ozalla, Enugu, Nigeria.

Tel: +234 8033248108

E-mail: snuwaezuoke@yahoo.com

Received: September 25, 2015

Accepted: October 09, 2015

Published: October 12, 2015

Citation: SN Uwaezuoke (2015) Prevention of Diabetic Nephropathy in Children and Adolescents: How Effective are the Current Strategies?. Int J Diabetol Vasc Dis Res, S5:001, 1-5. doi: http://dx.doi. org/10.19070/2328-353X-SI05001

Copyright: SN Uwaezuoke ${ }^{\odot}$ 2015. This is an open-access article distributed under the terms of the Creative Commons Attribution License, which permits unrestricted use, distribution and reproduction in any medium, provided the original author and source are credited.

\section{Introduction}

Type 1 diabetes mellitus (T1DM) is the predominant form of diabetes mellitus (DM) in children and adolescents [1], but the prevalence of Type 2 diabetes mellitus (T2DM) in these age groups is currently increasing worldwide [2]. The presence of persistent microalbuminuria in T1DM is strongly predictive of overt proteinuria (macroalbuminuria), subsequent end-stage renal failure and occasionally of cardiovascular disease $[2,3]$. On the other hand, T2DM is a major risk factor for cardiovascular events. Specifically, in patients with T1DM, hypertension and decline in renal function occur after the onset of macroalbuminuria; but in those with T2DM, hypertension and a decline in renal function may occur when there is microalbuminuria [2].

One of the major challenges of T1DM or even T2DM is the development of diabetic nephropathy (DN) [4]. DN is a clinical syndrome characterized by persistent albuminuria $(>300 \mathrm{mg} / \mathrm{d}$ or $200 \mu \mathrm{g} / \mathrm{min}$ ) confirmed on at least two occasions 3 to 6 months apart, progressive decline in glomerular filtration rate (GFR), and hypertension. DN is categorized into stages: microalbuminuria (urine albumin excretion of $>20 \mu \mathrm{g} / \mathrm{min}$ and $\leq 199 \mu \mathrm{g} /$ minor an albumin to creatinine ratio $(\mathrm{mg} / \mathrm{mmol})$ of 2.5 to 25 in males and 3.5 to 35 in females) [2], and macroalbuminuria (urine albumin excretion of $\geq 200 \mu \mathrm{g} / \mathrm{min}$ ). DN evolves over a long period of time-usually over 10 to 20 years-starting from microalbuminuria and progressing to end stage renal failure [1]. While microalbuminuria in children is reversible and may not therefore evolve to end-stage renal failure, macroalbuminuria inevitably progresses to end-stage renal failure irrespective of any known treatment [1].

Overt DN is thus very rare in the pediatric age group [5, 6], because renal involvement occurs in five stages which are related to the duration of T1DM [4, 5]. Stage 1 occurs with the onset of diabetes and is characterized by enlarged kidneys and hyperfiltration 
with increased GFR. Stage 2 occurs over 2 to 5 years; although similar to Stage 1, histopathologic abnormalities are however seen on renal biopsy. Stage 3 or incipient $\mathrm{DN}$ is characterized by microalbuminuria and normal renal function. With the onset of this stage about 5 years after diagnosis of diabetes, hypertension and progressive decline in GFR develop. Stage 4 or overt DN is the stage of dipstick positive proteinuria (macroalbuminuria) and rapid decline in GFR. Within 10 years of Stage 4, Stage 5 or end-stage renal failure sets in. Therefore, a consensus guideline recommends commencement of screening for microalbuminuria after 5 years of diabetes $[4,7,8]$.

Apart from the duration of diabetes, and normal glycaemic control, there are other risk factors which may influence the onset and progression of $\mathrm{DN}$. This paper aims to review these risk factors and the effectiveness of the current strategies for prevention of $\mathrm{DN}$ in childhood diabetes.

Literature search was conducted on Google and PubMed databases using appropriate search terms.

\section{Risk factors for diabetic nephropathy}

Although DN with consequent end-stage renal failure constitutes one of the leading causes of mortality in T1DM [5], overt DN is very uncommon in the paediatric age group [6]. However, one report described a 13 year-old female adolescent with poorly-controlled T1DM who developed overt DN only after 4 years of diabetes despite the absence of microvascular complications such as diabetic retinopathy [9]. Previous reports have also confirmed the possibility of overt DN before 5 years of diabetes in pre-pubertal, poorly-controlled patients with T1DM $[10,11]$. Conversely, renal function can be impaired even in T2DM patients with normoalbuminuria and microalbuminuria as well-known cardiovascular risk factors such as hypertension and arteriosclerosis appear to have a close relationship with renal damage in T2DM [12]. Consequently, some authors have suggested that screening for microalbuminuria in T1DM should be done at an earlier age than is currently recommended-especially in patients with identified risk factors for DN such as poor glycemic control and pubertal growth spurt [9]. Unlike in T1DM, where the strict glycemic control is the main preventive strategy for DN, in T2DM, the control of modifiable risk factors such as hypertension, hyperlipidemia, and obesity may assume priority [12]. In fact, some investigators have demonstrated a strong relationship between microalbuminuria and adolescent years, thus suggesting that adolescence and puberty are non-modifiable risk factors for progression of DN in T1DM [13]. Apart from hypertension and hyperlipidemia, smoking habits, albuminuria per se, as well as genetic predisposition are also known risk factors [5, 7, 14, 15]. Epidemiological [16] and familial studies [17-21] have demonstrated that genetic predisposition contributes to the development of $\mathrm{DN}$ in patients with both T1DM and T2DM. Additional findings supporting this observation include the report that diabetic siblings of patients with diabetes and renal disease are five times more likely to develop nephropathy than diabetic siblings of diabetic patients without renal disease [22], and the existence of a strong concordance of both nephropathy and renal histopathology in twins with T1DM [23]. In a study of Brazilian families with two or more diabetic members, the presence of diabetic nephropathy in the probands was significantly associated with a 3.75-fold increased risk of dia- betic nephropathy in the diabetic siblings [21].

Furthermore, low levels of high-density lipoprotein, low socioeconomic status, and male gender reportedly had significant association with $\mathrm{DN}$ in subjects with T2DM while the combination of blood pressure values in the high-normal range with moderately elevated levels of total cholesterol and hemoglobin A1c (a marker for average glycemic levels over the previous 3 months) defines a high-risk group for the progression to DN and for clinical events related to arteriosclerotic cardiovascular disease [24]. These observations are corroborated by a group of researchers in Thailand who documented duration of diabetes, HbA1c levels and uncontrolled hypertension as risk factors for DN in T2DM [25]. Admittedly, most of these studies on T2DM were conducted on adult subjects; nevertheless the findings could equally apply to children and adolescents among whom the global prevalence is rising [26].

\section{Diabetic nephropathy and microvascular compli- cations in T1DM and T2DM: the differences}

Patients with DN and T1DM almost always have other signs of diabetic microvascular disease, such as retinopathy and neuropathy. The retinopathy-which is easy to detect clinically-usually precedes the onset of overt DN in these patients. By the time advanced retinopathy has occurred, there are usually histologic changes in the glomeruli, as well as evidence of microalbuminuria. However, there are some patients with advanced retinopathy who have little or no renal disease as evaluated by renal biopsy and proteinuria. Rarely, T1DM may present with DN without preceding or associated retinopathy [9]. On the other hand, T2DM patients with marked proteinuria and retinopathy most likely have DN while those without retinopathy have a high incidence of non-diabetic glomerular disease.

Glomerular hyperperfusion is among the earliest changes demonstrable in DN-which is accompanied by microalbuminuria. Microalbuminuria not only serves as a sensitive early indicator of $\mathrm{DN}$ but also a powerful predictor of its subsequent progression. Eighty percent of T1DM patients with microalbuminuria will progress to overt nephropathy within 10-15 years. Of these patients, 50\% will develop ESRD within 10 years and $75 \%$ within 20 years in the absence of specific interventions [27]. Among patients with T2DM, $20-40 \%$ of patients with microalbuminuria will progress to overt nephropathy, though only $20 \%$ of those patients will go on to ESRD within the next 20 years [27]. Microalbuminuria is also a powerful predictor of cardiovascular disease in both T1DM and T2DM. Thus, in T1DM, yearly screening should begin after puberty and 5 years after initial diagnosis. In T2DM, yearly screening for microalbuminuria should begin at the time of diagnosis because of the likelihood that diabetes has been present for several years by the time it is diagnosed.

\section{Prevention and treatment strategies for diabetic nephropathy}

Both tight glycemic control and intensive control of elevated blood pressure have significant impact on prevention and progression of DN [28]. In general, the goal for glycemic control is a blood glucose level as close to normal ( HbA1c $<7 \%$ ) as possible without causing dangerous hypoglycemia [29]. The importance of 
prevention cannot be overemphasized; once overt nephropathy is present, progression cannot be halted but only retarded. It is much more effective to screen for early nephropathy with sensitive tests for microalbuminuria and to prevent or halt the earliest stages of renal damage by tight glycemic control and control of hypertension [29].

From several studies, tight glycemic control has been shown to decrease the risk of microvascular disease in both T1DM and T2DM [14, 30-32]. It is well established that poor glycemic control is critical in the etiology of diabetic nephropathy. For instance, $\mathrm{DN}$ is rare in patients with $\mathrm{HbA} 1 \mathrm{c}$ consistently $<7.5-8.0 \%[4,14]$.

In the Diabetes Control and Complications Trial (DCCT), tight glycemic control in patients with T1DM reduced the incidence of microalbuminuria by $39 \%$ in the primary prevention group and reduced the progression from microalbuminuria to macroalbuminuria by $54 \%$ in the secondary prevention group [30]. Furthermore, in the United Kingdom Prospective Diabetes Study (UKPDS), there was a $34 \%$ decrease in the risk of microalbuminuria in patients with T2DM treated more intensively for glycemic control [31]. Thus, there is a strong clinical evidence for the effectiveness of tight glycemic control in the prevention of $\mathrm{DN}$.

Secondly, hypertension is a well established contributory cause of diabetic microvascular complications [33], and its control decreases albuminuria, delays nephropathy, and improves survival in both T1DM and T2DM [34]. Notably, the renin-angiotensin system is the target of the most effective strategy for both control of hypertension and, independently, for reduction of the pathophysiologic abnormalities which result in proteinuria [35]. This is best established in T1DM, but there is increasing evidence that the same pathophysiologic principles and treatment also apply in T2DM [33]. In addition, hypertension may be a common primary risk for the renal and other nonrenal cardiovascular complications of diabetes [36, 37]. One review has noted that apart from good glycemic control, antihypertensive treatment especially with angiotensin converting enzyme (ACE) inhibitors, often combined with other agents is quite effective in preventing progression of $\mathrm{DN}$ in all its stages [38]. In T2DM, hypertension is often an early finding, and blood pressure significantly increases according to the degree of albuminuria, normo-microalbuminuria and clinical proteinuria (macroalbuminuria) [38]. Hypertension is an important risk for both DN and cardiovascular disease. The author further notes that based on several studies, antihypertensive treatment - particularly with ACE-inhibitors-is important in both preventing and treating microalbuminuria, and thus preventing progression to overt DN [38]. Indeed, there is accumulating evidence to suggest that the use of antihypertensive agents which target the renin-angiotensin system can retard the progression of $\mathrm{DN}$, as well as provide cardioprotection in patients with T2DM and microalbuminuria [2]. Antihypertensive treatment in patients with microalbuminuria and T2DM should therefore be initiated with angiotensin converting enzyme (ACE) inhibitors or angiotensin-II type 1 receptor blockers [2]. Nevertheless, neither ACE inhibitor nor ARB is currently recommended in normotensive, normoalbuminuric diabetics for primary prevention of $\mathrm{DN}$.

Currently, most evidence and published guidelines suggest ACE inhibitors as first-choice antihypertensives in patients with diabetes $[27,39]$. Notably, the degree of blood-pressure reduction rather than the class of antihypertensive agent used appears to be the most important factor in renoprotection. Calcium-channel blockers have been demonstrated to have beneficial effects [40] and combinations of ACE inhibition and calcium-channel blockade have shown positive results [41].

Dyslipidemia is also one of the putative risk factors for $\mathrm{DN}$ in patients with diabetes [42]. In addition, it is known to hasten the atherosclerotic process and is thus one of the major risk factors for cardiovascular disease in diabetes. A recent strategy is the use of nutraceuticals and functional foods which have been reported to reduce the overall cardiovascular risk induced by dyslipidemia by acting in synergy with statins [43]. Nutraceuticals refer to foods or food components which provide medical benefits including prevention and/or treatment of disease while functional foods are any foods or food ingredients which may provide health benefit beyond the traditional nutrients they contain [43]. Furthermore, dietary omega-3 polyunsaturated fatty acids could improve endothelial function and structure early in life, and therefore reduce cardiovascular risk profile later in life since endothelial dysfunction is an early marker of atherosclerosis [44].

The diabetic milieu is a complex environment where a number of interventions may be utilized to target various pathological processes. Given that no single therapy completely ameliorates DN, novel strategies are needed to complement existing interventions [45]. Some of these novel agents include the following; allopurinol -uric acid antagonist-(which improves endothelial dysfunction and reduces urinary TGF- $\beta$ in DN) [46-48], vitamin D (which reduces albuminuria) [49], renin inhibitors such as aliskiren (which lowers blood pressure and reduces albuminuria) [50], and endothelial inhibitors such as atrasentan (which also lowers blood pressure and reduces albuminuria) [51].

Finally, high dietary protein has renal hemodynamic effects that include increased glomerular filtration rate (GFR), hyperfiltration, and increased intraglomerular pressure which are probably accentuated by poor glycemic control [29]. Dietary protein restriction has been shown to decrease renal functional deterioration in both T1DM and T2DM [52, 53]. For example, in a 5-year prospective study of patients with T1DM, those on a protein- and phosphaterestricted diet showed a decline of GFR of only $0.26 \mathrm{ml} / \mathrm{min} /$ month compared with $1.01 \mathrm{ml} / \mathrm{min} /$ month in those on unrestricted diets [54].

Current recommendations are for dietary protein at the level of the Recommended Dietary Allowance of $0.8 \mathrm{~g} / \mathrm{kg} /$ day, accounting for $10 \%$ of total calories. In selected patients with decreasing GFR, it may be useful to decrease the prescribed protein intake to $0.6 \mathrm{~g} / \mathrm{kg} /$ day as directed by a professional nutritionist [29].

\section{Conclusion}

Among the microvascular complications of diabetes in children and adolescents, DN remains the most important contributor of mortality. DN evolves over a long period of time-usually over 10 to 20 years- starting from microalbuminuria and progressing to end stage renal failure. Both tight glycemic control and intensive control of hypertension have significant impact on prevention and progression of DN. While tight glycemic control in patients with T1DM reduces the incidence of microalbuminuria and the 
progression from microalbuminuria to macroalbuminuria, there is overwhelming evidence to show that antihypertensive treatment particularly with ACE-inhibitors- is important in both preventing and treating microalbuminuria, and thus preventing progression to overt DN. Dietary protein restriction is also a useful adjunct strategy in retarding the progression of $\mathrm{DN}$. Based on evidence in the literature, the current strategies for the prevention of DN in childhood diabetes are still effective and should remain part of the standard management guidelines. Other novel therapeutic strategies have been recently tried and found potentially effective. Research is ongoing to establish the clinical efficacy and usefulness of some of these new agents in future.

\section{References}

[1]. Koulouridis E (2001) Diabetic nephropathy in children and adolescents and its consequences in adults. J Pediatr Endocrinol Metab 14(Suppl 5): S13671377.

[2]. Jerums G, MacIsaac RJ (2002) Treatment of microalbuminuria in patients with type 2 diabetes mellitus. Treat Endocrinol 1(3): 163-173.

[3]. McKenna K, Thompson C (1997) Microalbuminuria: a marker to increased renal and cardiovascular risk in diabetes mellitus. Scott Med J 42(4): 99-104.

[4]. Deferrari G, Repetto M, Calvi C, Ciabattoni M, Rossi C, et al. (1998) Diabetic nephropathy: from micro-to macroalbuminuria. Nephrol Dial Transplant 13(Suppl 8): 11-15.

[5]. Feld LG (1999) Diabetic Nephropathy. In Pediatric Nephrology. (4 ${ }^{\text {th }}$ edtn), Lippincott Williams and Wilkins, Baltimore. 633-640.

[6]. Danne T, Kordonouri O, Hovener G, Weber B (1997) Diabetic angiopathy in children. Diabet Med 14(12): 1012-1025.

[7]. Bogdanovic R (2001) Diabetic nephropathy in children. Nephrol Dial Transplant 16(Suppl 6): S120-122.

[8]. Consensus Guidelines (2000). Diabetic Disease. ISAPD Consensus Guidelines for the management of Type 1 Diabetes Mellitus in children and adolescents. Published by the Medical Forum International. 99.

[9]. Maghribi H, Abu-Odeh A (2006) Early diabetic nephropathy in a paediatric patient. JRMS 13(1): 51-53.

[10]. Francis J, Rose SJ, Raafat F, Milford DV (1997) Early onset of diabetic nephropathy. Arch Dis Child 77(6): 524-525.

[11]. DeClue TJ, Campos A (1994) Diabetic nephropathy in a prepubertal diabetic female. J Pediatr Endocrinol 7(1): 43-46.

[12]. Molnár M, Wittmann I, Nagy J (2000) Prevalence, course and risk factors of diabetic nephropathy in type-2 diabetes mellitus. Med Sci Monit 6(5): 929-936.

[13]. Twyman S, Rowe D, Mansell P, Schapira D, Betts P, et al. (2001) Longitudinal study of urinary albumin excretion in young diabetic patients- Wessex Diabetic Nephropathy Project. Diabet Med 18(5): 402-408.

[14]. Di Landro D, Catalano C, Lambertini D, Bordin V, Fabbian F, et al. (1998) The effect of metabolic control on development and progression of diabetic nephropathy. Nephrol Dial Transplant 13(Suppl 8): S35-43.

[15]. Sochett E, Daneman D (1999) Early diabetes-related complication in children and adolescents with type 1 diabetes. Implications for screening and intervention. Endocrinol Metab Clin North Am 28(4): 865-882.

[16]. Krolewski AS, Warram JH, Christlieb AR, Busick EJ, Kahn CR (1985) The changing natural history of nephropathy in type I diabetes. Am J Med 78(5): 785-794.

[17]. Quinn M, Angelico MC, Warram JH, Krolewski AS (1996) Familial factors determine the development of diabetic nephropathy in patients with IDDM. Diabetologia 39(8): 940-945.

[18]. Pettitt DJ, Saad MF, Bennett PH, Nelson RG, Knowler WC (1990) Familial predisposition to renal disease in two generations of Pima Indians with type 2 (non-insulin-dependent) diabetes mellitus. Diabetologia 33(7): 438-443.

[19]. Freedman BI, Tuttle AB, Spray BJ (1995) Familial predisposition to nephropathy in African-Americans with non-insulin-dependent diabetes mellitus. Am J Kidney Dis 25(5): 710-713.

[20]. The Diabetes Control and Complications Trial Research Group (1997) Clustering of long-term complications in families with diabetes in the diabetes control and complications trial. Diabetes 46(11): 1829-1839.

[21]. Canani LH, Gerchman F, Gross JL (1999) Familial clustering of diabetic nephropathy in Brazilian Type 2 diabetic patients. Diabetes 48(4): 909-913.

[22]. Seaquist E, Goetz F, Rich S, Barbosa J (1989) Familial clustering of diabetic kidney disease: evidence for genetic susceptibility to diabetic nephropathy. $\mathrm{N}$ Engl J Med 320(18): 1161-1165.

[23]. Fioretto P, Steffes MW, Barbosa J, Rich SS, Miller ME, et al. (1999) Is diabetic nephropathy inherited? Studies of glomerular structure in type 1 diabetic sibling pairs. Diabetes 48(4): 865-869.
[24]. Ravid M, Brosh D, Ravid-Safran D, Levy Z, Rachmani R (1998) Main risk factors for nephropathy in type 2 diabetes mellitus are plasma cholesterol levels, mean blood pressure, and hyperglycemia. Arch Intern Med 158(9): 998-1004.

[25]. Krairittichai U, Potisat S, Jongsareejit A, Sattaputh C (2011) Prevalence and risk factors of diabetic nephropathy among Thai patients with type 2 diabetes mellitus. J Med Assoc Thai 94(Suppl 2): S1-5.

[26]. Hu FB (2011) Globalization of diabetes: the role of diet, lifestyle, and genes. Diabetes Care 34(6): 1249-1257.

[27]. American Diabetes Association (1999). Position statement: Diabetic nephropathy. Diabetes Care 22(Suppl 1): S66-69.

[28]. O'Connor PJ, Spann SJ, Woolf SH (1998) Care of adults with type 2 diabetes mellitus: a review of the evidence. J Fam Pract $47(5$ Suppl): S13-22.

[29]. Evans TC, Capell P (2000) Diabetic Nephropathy. Clinical Diabet 18(1): $1-16$

[30]. The Diabetes Control and Complications Trial Research Group (1993). The effect of intensive treatment of diabetes on the development and progression of long-term complications in insulin-dependent diabetes mellitus. N Engl J Med 329: 977-986.

[31]. UK Prospective Diabetes Study Group (1998) Intensive blood-glucose control with sulphonylureas or insulin compared with conventional treatment and risk of complications in patients with type 2 diabetes (UKPDS 33). Lancet 352: 837-853.

[32]. Molitch ME (1997) The relationship between glucose control and the development of diabetic nephropathy in type I diabetes. Sem Nephrol 17(2): 101-113.

[33]. Luno J, Garcia de Vinuesa S, Gomez-Campdera F, Lorenzo I, Valderrabano F (1998) Effects of antihypertensive therapy on progression of diabetic nephropathy. Kidney Int Suppl 68: S112-119.

[34]. Parving HH (1998) Is antihypertensive treatment the same for NIDDM and IDDM patients? Diabetes Res Clin Pract 39(Suppl): S43-47.

[35]. Ritz E, Miltenberger-Miltenyi G, Wagner J, Rychlik I (1998) Diabetes-renal function--what are the special problems? Basic Res Cardiol 93(Suppl 2): $125-130$.

[36]. Ritz E, Rychlik I, Miltenberger-Miltenyi G (1998) Optimizing antihypertensive therapy in patients with diabetic nephropathy. J Hypertens Suppl 16: S17-22.

[37]. Nosadini R, Abaterusso C, Dalla Vestra M, Bortoloso E, Saller A, et al. (1998) Efficacy of antihypertensive therapy in decreasing renal and cardiovascular complications in diabetes mellitus. Nephrol Dial Transplant 13(Suppl 8): 44-48.

[38]. Mogensen CE (1999) Drug treatment for hypertensive patients in special situations: diabetes and hypertension. Clin Exp Hypertens 21(5-6): 895-906.

[39]. Mogensen CE (1999) Microalbuminuria, blood pressure and diabetic renal disease: origin and development of ideas. Diabetologia 42(3): 263-285.

[40]. Rossing P (1998) Promotion, prediction and prevention of progression of nephropathy in Type 1 diabetes mellitus. Diabet Med 15(11): 900-919.

[41]. Bakris GL, Weir MR, DeQuattro V, McMahon FG (1998) Effects of an ACE inhibitor/calcium antagonist combination on proteinuria in diabetic nephropathy. Kidney Int 54(4): 1283-1289.

[42]. Chaturvedi N, Fuller JH, Taskinen MR (2001) Differing associations of lipid and lipoprotein disturbances with the macrovascular and microvascular complications of Type 1 diabetes. Diabetes Care 24(12): 2071-2077.

[43]. Scicchitano P, Cameli M, Maiello M, Modesti PA, Muiesan ML, et al. (2014) Nutraceuticals and dyslipidemia: Beyond the common therapeutics. J Funct Foods 6: 11-32.

[44]. Ciccone MM, Scicchitano P, Gesualdo M, Zito A, Carbonara S, et al. (2013) The role of omega- 3 polyunsaturated fatty acids supplementation in childhood: a review. Recent Pat Cardiovasc Drug Discov 8(1): 42-55.

[45]. Lim AKH (2014) Diabetic nephropathy: complications and treatment. Int ] Nephrol Renovasc Dis 7: 361-381.

[46]. Dogan A, Yarlioglues M, Kaya MG, Karadag Z, Dogan S, et al. (2011) Effect of long-term and high-dose allopurinol therapy on endothelial function in normotensive diabetic patients. Blood Press 20(3): 182-187.

[47]. Butler R, Morris AD, Belch JJ, Hill A, Struthers AD (2000) Allopurinol normalizes endothelial dysfunction in Type 2 diabetics with mild hypertension. Hypertension 35(3): 746-751.

[48]. Talaat KM, el-Sheikh AR (2007) The effect of mild hyperuricemia on urinary transforming growth factor beta and the progression of chronic kidney disease. Am J Nephrol 27(5): 435-440.

[49]. Nakai K, Fujii H, Kono K, Goto S, Kitazawa R, et al. (2014) Vitamin D activates the Nrf2-Keap1 antioxidant pathway and ameliorates nephropathy in diabetic rats. Am J Hypertens 27(4): 586-595.

[50]. Persson F, Rossing P, Schjoedt KJ, Juhl T, Tarnow L, et al. (2008) Time course of the antiproteinuric and antihypertensive effects of direct renin inhibition in Type 2 diabetes. Kidney Int 73(12): 1419-1425.

[51]. de Zeeuw D, Coll B, Andress D, Brennan JJ, Tang H, et al. (2014) The endothelin antagonist atrasentan lowers residual albuminuria in patients with 
Type 2 diabetic nephropathy. J Am Soc Nephrol 25(5): 1083-1093.

[52]. Barsotti G, Cupisti A, Barsotti M, Sposini S, Palmieri D, et al. (1998) Dietary treatment of diabetic nephropathy with chronic renal failure. Nephrol Dial Transplant 13(Suppl 8): 49-52.

[53]. Toeller M, Buyken AE (1998) Protein intake--new evidence for its role in diabetic nephropathy. Nephrol Dial Transplant 13(8): 1926-1927.

[54]. Zeller K, Whittaker E, Sullivan L, Raskin P, Jacobson HR (1991) Effect of restricting dietary protein on the progression of renal failure in patients with insulin-dependent diabetes mellitus. N Engl J Med 324(2): 78-84.
Special Issue on

"Diabetes and Nephrology"

Edited by:

Dr. Ayman Maher Nagib, Consultant of nephrology, Urology and nephrology center, Mansoura University, Egypt.

E-mail: ayman_maher2005@yahoo.com 